\title{
Situación actual de la hemodiálisis domiciliaria
}

\author{
Dolores Andreu Périz, Mª Carmen Moreno Arroyo, Miguel Ángel Hidalgo Blanco
}

Facultad de Medicina y Ciencias de la Salud. Universidad de Barcelona. España

La hemodiálisis domiciliaria (HDD) es la forma de tratamiento de depuración renal extracorpórea en la que el paciente realiza por sí mismo la técnica en su propia casa. Los requisitos para incluir a un paciente en HDD además de que este lo considere su mejor opción, son que se mantenga razonablemente estable durante la sesión depuradora, un buen acceso vascular y en la medida del posible un espacio exclusivo para el monitor y el utillaje además de la presencia de un acompañante durante el tratamiento. Las ventajas son muy numerosas: evitar el contacto con un ambiente hospitalario y darle mayor autonomía parecen razones suficientes para considerar a la HDD como una primera elección ${ }^{1}$. Esta forma de tratamiento se practica desde los inicios de los programas de diálisis hospitalaria, en los que la falta de infraestructuras llevó a los pioneros de la nefrología a crear estrategias para dar respuestas a la creciente demanda de puestos de diálisis. En Estados Unidos e Inglaterra llegaron a incluirse en HDD a miles de pacientes y parecía que sería la mejor manera de abaratar los costes y dar a estas personas una la mejor opción de tratamiento².

Numerosas circunstancias justifican que la HDD no se haya tenido el desarrollo esperado, entre ellas destacaría el éxito de los trasplantes, el auge de la diálisis peritoneal, técnica mucho más sencilla que también se practica a domicilio, pero sobre todo las características de muchos pacientes que inician tratamiento y que cada vez son más añosos y con mayor morbilidad².

Sin embargo, en los últimos años nuevas formas de prescripción de la hemodiálisis en cuanto a tiempo y frecuencia están obteniendo excelentes resultados y la

$$
\begin{gathered}
\text { Correspondencia: } \\
\text { Dolores Andreu Periz } \\
\text { Campus de Bellvitge. Universidad de Barcelona } \\
\text { C/ Feixa Llarga, s/n } \\
08907 \text { L'Hospitalet de Llobregat. Barcelona } \\
\text { E-mail:Iolaandreu@ub.edu }
\end{gathered}
$$

evidencia demuestra que con sesiones más frecuentes y/o más prolongadas se alcanza un mayor control clínico y mejor calidad de vida ${ }^{3}$. Estas nuevas formas de tratamiento son mucho más tolerables para el paciente y permiten administrar con más eficiencia los recursos disponibles si se pueden practicar a domicilio, evitando los desplazamientos a un centro y facilitando la elección de horarios ${ }^{3}$. Estas razones hacen que la HDD esté creciendo de manera sustancial en los países desarrollados aunque, la prevalencia en España sigue siendo muy baja y apenas alcanza al 0,2\% de la población en diálisis ${ }^{4}$. Numerosas iniciativas intentan revertir esta situación, entre otras destacaría el apoyo de la SEN que cuenta con un grupo de trabajo referido a esta técnica 4 .

Esta situación de auge de la HDD aconseja revisar las últimas publicaciones sobre el tema en los que se constata las indudables ventajas que ofrece a HDD pero también las dificultades de su implementación. El seguimiento de los pacientes tras un evento que requiera ingreso hospitalario, las complicaciones que sufren los pacientes, especialmente las derivadas de problemas en el acceso vascular y las vivencias de los pacientes y su familia se recogen en los artículos que se han seleccionado a continuación.

Raphael MJ, Nadeau-Fredette AC, Tennankore KK, Chan CT. A virtual ward for home hemodialysis patients - a pilot trial. Can J Kidney Health Dis. 2015 Nov 1; 2:37.

En este estudio se plante el problema del seguimiento que se realiza a los pacientes renales tras ser dados de alta de un ingreso hospitalario. En este período aparece el riesgo de que haya un vacío entre la atención que reciben durante la hospitalización y la que va a recibir en el domicilio. Para intentar paliar esta problemática en varios países se ha propuesto utilizar estrategias como la Virtual Ward (VW) o sala de hospitalización virtual, modelo en el que un equipo multidisciplinar pretende proporcionar después del alta hospitalaria y a corto plazo una mejor atención 
a los pacientes y paliar en lo posible los déficits de atención que puedan encontrar en el domicilio.

Además de la hospitalización los pacientes con enfermedad renal plantean problemas como la necesidad frecuente de realizar procedimientos médicos hospitalarios y el estar sometidos a tratamientos farmacológicos complejos por lo que podrían ser candidatos a un seguimiento mediante una VW. Con el objetivo de evaluar la viabilidad de la implementación de una VW para pacientes en HDD tras un ingreso hospitalario o en circunstancias en las que se requiera un procedimiento clínico específico, en este trabajo se describe un estudio piloto que incluyó a pacientes en HDD que cumplieran uno o más de los cuatro criterios de inclusión:

\section{- Alta hospitalaria \\ - Realización de un procedimiento médico en el hospital \\ - Prescripción de un antibiótico \\ - Finalización de la formación para hacer hemo- diálisis en casa}

Este estudio se llevó a cabo en un hospital universitario de Toronto en el periodo 2012-2013 y se realizó un seguimiento durante 14 días a pacientes en HDD. Se evaluaron las entrevistas telefónicas realizadas por los clínicos sobre tres posibles necesidades relacionadas con: a) Cambios en la prescripción de hemodiálisis, b) Coordinación de la atención de seguimiento y c) Necesidad de cambio de medicación. Se realizaron mediante la VW, 52 actuaciones a 35 pacientes en programa de HDD y se identificó en cada caso el número de situaciones de riesgo que podrían provocar una potencial falta de atención, también se identificaron posibles predictores de este riesgo, así como los eventos negativos que requirieron de ingreso hospitalario, visita a urgencias o a la unidad de diálisis hospitalaria.

La implementación y ejecución del estudio piloto sobre la eficacia de la VW en pacientes sometidos a HDD demostraron que esta estrategia es técnicamente factible y práctica. Una posible falta de atención se identificó en $35(67 \%)$ de las actuaciones llevadas a cabo mediante la VW y se llevaron a cabo 85 actuaciones para evitar eventos negativos. No hubo características demográficas que permitieran predecir un déficit de atención. En el periodo estudiado hubo 9 reingresos, 13 visitas al servicio de urgencias, 7 visitas no programadas a la unidad de hemodiálisis del hospital. Aunque los resultados de este estudio están limitados por el pequeño tamaño de la muestra y la experiencia de un solo centro los autores llegan a la conclusión que la implementación de una sala virtual para pacientes con HDD es práctica y viable e identifica muchas lagunas en la atención clínica que podrían producir eventos adversos, por lo que abogan por la necesidad de hacer un estudio prospectivo amplio y multicéntrico que corroborara estas afirmaciones.

\section{Combes G, Allen K, Sein K, Girling A, Lilford R. Taking hospital treatments home: a mixed methods case study looking at the barriers and success factors for home dialysis treatment and the influence of a target on uptake rates. Implement Sci. 2015 Oct 27; 10: 148.}

Los autores de este estudio ratifican el hecho de que a pesar de las políticas de salud y las pruebas que promueven la diálisis domiciliaria las tasas de pacientes en esta técnica han estado cayendo durante más de 10 años en Inglaterra, país en los que tradicionalmente se ha apostado por la HDD. Ante el hecho de que 2010 las autoridades sanitarias de la región de West Midlands decidieran implementar unas políticas para incrementar los tratamientos domiciliarios, en este trabajo se plantea analizar como los hospitales de la región abordaron dichas políticas. Los cambios cuantitativos en el incremento de los tratamientos a domicilio en siete hospitales de la región de West Midlands se compararon con los del resto de Inglaterra durante 3 años, llevándose a cabo este estudio mediante un modelo de regresión logística y entrevistas cualitativas que se realizaron en cuatro hospitales a 96 miembros del personal clínico o de gestión y a 93 pacientes en programa de diálisis. Se exploró el impacto, las dificultades y los elementos facilitadores que habían surgido durante el periodo estudiado.

Los tratamientos domiciliarios aumentaron significativamente en los siete hospitales del estudio en comparación con los 3 años anteriores a la introducción de las nuevas políticas sanitarias y con el resto hospitales de Inglaterra, donde no se incrementaron estas formas de tratamiento. Los cuatro factores principales que justificaron el desarrollo del tratamiento domiciliario fueron: la propuesta de las autoridades sanitarias que se vinculó a sanciones económicas; una financiación adicional para el personal y equipo especializado; definir los cometidos y visualizar las mejorías clínicas, además de tener buenos sistemas de formación y apoyo al paciente. Las tres dificultades principales para la consecución de un incremento de las diálisis domiciliarias fueron: falta de formación para el personal no especializado, la educación insufi- 
ciente para el paciente y no considerar sus necesidades emocionales o psicológicas.

Las conclusiones del estudio muestran el impacto de los objetivos de la administración, que incluyan sanciones económicas, para lograr un cambio y destacan cómo los hospitales pueden aumentar significativamente los tratamientos domiciliarios y los autocuidados proporcionando indicaciones útiles para limitar los principales obstáculos y diseñando elementos facilitadores. Otro aspecto relevante de estas conclusiones es que hay dos áreas que necesitan mejorar para atender adecuadamente a los pacientes: la educación individualizada y la prestación de un apoyo emocional continuo.

Bi SH, Tang W, Rigodanzo-Massey N, Young BA, Blagg CR, Wang T, Ahmad S. Infection-Related Hospitalizations in Home Hemodialysis Patients. Blood Purif. 2015; 40(3):187-93.

Puesto que una de las razones que han llevado al auge de la HDD es la mayor facilidad para utilizar diferentes modalidades de hemodiálisis los autores de este trabajo se plantean que es importante conocer no solo los problemas que sufren estos pacientes sino también si se pueden relacionar con estas variantes de tratamiento. Se sabe que las infecciones son una causa frecuente de hospitalización en los pacientes en hemodiálisis, no obstante hay pocos estudios sobre eventos infecciosos en pacientes que hagan la técnica en su hogar. En este estudio los autores se pretenden examinar las características de las infecciones que requirieron hospitalización o no de paciente en HDD y la relación entre la modalidad de diálisis y la aparición de infecciones.

El estudio se llevó a cabo entre julio de 2005 y agosto de 2010 y se obtuvieron los datos a partir de un sistema informático centralizado. Se registraron las características de 165 pacientes (113 mujeres) con una media de edad de 50,8 $\pm 15,6$ años que estaban en diálisis $70,8 \pm 77,9$ meses. La modalidad de tratamiento fue de diálisis $5-6$ veces semana $(66,7 \%)$, diálisis nocturna diaria $(9,1 \%)$ y diálisis convencional de 3 veces por semana $(24,2 \%)$.

En el periodo estudiado se observaron eventos infecciosos en 134 pacientes que requirieron de 176 ingresos hospitalarios, de estos, $49(40,6 \%)$ se relacionaron con la infección lo que supuso una tasa global de 5,94 eventos / 100 personas-año. La primera causa $(38,5 \%)$ de hospitalización fue la infección, la segunda los problemas quirúrgicos y la tercera la disfunción del acceso vascular. El $21,8 \%$ de los pacientes tenían al menos 1 hospitalización relacionada con la infección y se identificó una amplia gama de infecciones, incluyendo sepsis, infecciones relacionadas con el acceso vascular, con el aparato respiratorio, con problemas gastrointestinales e infecciones de tejidos blandos. Aunque la infección fue la primera causa de ingreso de los pacientes que tenía problemas con el acceso vascular la tasa de infecciones no relacionadas con el acceso vascular supero a la de infecciones de dichos accesos (1,7: 1). La edad, la raza, la etiología y la diabetes mellitus fue similar entre los pacientes que presentando una infección requirieron ser hospitalizados y los que no. El porcentaje de paciente en diálisis diaria que requirió ser hospitalizado por la infección fue menor que en el grupo sin hospitalización. Sin embargo el modelo de regresión de Cox no asoció un aumento de riesgo de infección por el uso del tipo de diálisis y solo se relacionó la diabetes mellitus con un aumento de los eventos relacionados con las infecciones. Estos datos permiten concluir a los autores que los eventos infecciosos que requieren de hospitalización son frecuentes en los pacientes en HDD y proponen mejorar en el conocimiento de las características de estas infecciones e incrementar las medidas preventivas para evitarlas.

Spry LA, Burkart JM, Holcroft C, Mortier L, Glickman JD. Survey of home hemodialysis patients and nursing staff regarding vascular access use and care. Hemodial Int 2015; 19: 225-34.

Abundando en la problemática de los eventos infecciosos de los pacientes en HDD en este artículo se aborda el estudio de las infecciones de los accesos vasculares que según sus autores causan más del $10 \%$ de los ingresos hospitalarios que en muchos casos obligan a reingresos tempranos. Las guías de buena práctica son difíciles de aplicar en la HDD, técnica en la que el paciente se autopunciona y además ante la actual tendencia de incrementar la dosis de diálisis suele recibir el tratamiento con mucha frecuencia. Se plantean en el estudio la hipótesis de que las pautas de entrenamiento para el manejo del acceso vascular no son homogéneas y como resultados los pacientes no cumplen lo establecido por las guías de buena práctica.

El objetivo de este estudio fue recopilar información sobre aspectos relacionados con el acceso vascular que permitieran analizar como manejaban sus accesos los pacientes en HDD. Como referencia de cuáles 
son las pautas adecuadas se utilizaron entre otras las recomendaciones de la American Nephrology Nurses Association y de la Kidney Disease Outcomes Quality Initiative. Se diseñaron dos cuestionarios, uno sobre la formación proporcionada a los pacientes y otro sobre las prácticas reales realizadas por los pacientes. En estos cuestionarios se hizo especial referencia a la evaluación de la formación, los métodos de cuidado, el uso de tecnología apropiada y el conocimiento y forma de actuar de los pacientes ante sintomatología de infección. Para asegurar que las preguntas estaban correctamente formuladas los cuestionarios se revisaron por cuatro grupos de enfermeras que formaban a pacientes en HDD, un grupo focal formado por siete pacientes y familiares y diez cuestionarios se cumplimentaron mediante entrevistas telefónicas a pacientes que no se incluyeron en los resultados finales.

Se distribuyeron las encuestas a 301 pacientes y a 55 enfermeras de 19 centros seleccionados al azar siendo la tasa de respuestas del $43 \%$ de los pacientes y el $76 \%$ de enfermeras. De los resultados obtenidos cabe destacar que las enfermeras no instruían uniformemente a los pacientes de acuerdo con las guías de buena práctica y los pacientes no seguían las recomendaciones de las enfermeras en aspectos como el uso de mascarilla, la forma de fijar las agujas, el retirar los apósitos de las punciones o el uso de antibióticos locales. Se entrenaba a los pacientes para reconocer los signos de infección pero estos no siempre lo comunicaban con la suficiente rapidez. Ningún paciente o enfermera afirmaron cumplir con todas las medidas recomendadas por las guías. La conclusión de este estudio es que es necesario estudiar el impacto que tiene en la aparición de infecciones las medidas que toman los pacientes en HDD para el cuidado de los accesos vasculares y que deben implementarse estrategias que permitan adaptar las guías de buena práctica para estos pacientes.

\begin{abstract}
Walker RC, Morton RL, Tong A, Marshall MR, Palmer S, Howard K. Patient and caregiver preferences for home dialysis-the home first study: a protocol for qualitative interviews and discrete choice experiments. BMJ Open. 2015 Apr 15; 5 (4).
\end{abstract}

Walker RC, Howard K, Morton RL, Palmer S, Marshall M, Tong A. Patient and Caregiver Values, Beliefs and Experiences When Considering Home Dialysis as a Treatment Option A Semi-Structured Interview Stud. Nephrol Dial Transplant. 2016; 31 (1): 133-141.
En la primera de estas dos publicaciones los autores exponen su propósito de llevar a cabo un estudio en Nueva Zelanda sobre un tema poco explorado: el punto de vista de los pacientes y sus cuidadores sobre la $H D D$, para esto dan a su proyecto un enfoque cualitativo con el objetivo de describir las preferencias y las opiniones de pacientes y cuidadores acerca de los factores que influyen en la elección del domicilio o el centro sanitario para llevar a cabo la diálisis De una población potencial de 150 pacientes y sus cuidadores se propusieron llevar a cabo entrevistas semiestructuradas a entre 30-40 pacientes más unos $10-15$ cuidadores mediante un modelo de elección discreta*.

La necesidad de profundizar en las causas por las que un paciente elige una u otra forma de tratamiento es en sí un tema importante, pero lo es más para los autores de este estudio pues en Nueva Zelanda, al igual que Australia, siempre han habido tasas de HDD superiores a las de otros países, Como ejemplo en 2012, en Nueva Zelanda se trataban con esta técnica al $19 \%$ de los pacientes y en Australia al 9\% mientras que en países como Canadá o Inglaterra estas cifras oscilan entre un 3- 5\%.

En el segundo trabajo los autores presentan sus resultados y conclusiones, finalmente obtuvieron una muestra de 43 pacientes y 9 cuidadores procedentes de 3 unidades de nefrología. De todos los paciente 18 estaban en situación de prediálisis, 4 en HDD, 13 en diálisis peritoneal y 9 en diálisis asistida. Tras el análisis de las entrevistas se identificaron las siguientes categorías:

a) Carece de poder de decisión: recibir una información compleja, sentirse impotente y presionado por la elección.

b) Las relaciones con el entorno: el estar mediatizadas por la influencia de la cultura, la familia, la confianza en los médicos, el aislamiento social.

c) Los cambios en el estilo de vida: mantener el empleo, evitar los traslados, considerar los gastos adicionales, buscar horarios flexibles, y garantizar tiempo libre.

d) Confianza en la elección de la forma de tratamiento: garantizar la seguridad, en función de la seguridad del profesional, el apoyo por parte de los compañeros, superar el miedo y mejorar la supervivencia.

Como conclusión de su trabajo los autores afirman que para fomentar la diálisis en el hogar es necesario dar mayor énfasis a los programas educativos centrados en el paciente. Un mayor conocimiento sobre la 
salud y considerar valores sociales y culturales pueden reducir los temores y dar confianza en la toma de decisiones. Los gastos que ocasiona el tratamiento en el domicilio se pueden minimizar mediante programas de reembolso, también apoyar el empleo y darles una ayuda económica adicional, especialmente a pacientes que viven en lugares alejados, favorecería la decisión de elegir un tratamiento domiciliario.

${ }^{*}$ El modelo de elección discreta es muy utilizado en estudios económicos y resulta apropiado cuando el objetivo no es predecir el comportamiento medio sino analizar los factores determinantes de la probabilidad de que un agente económico individual elija un curso de acción dentro de un conjunto, generalmente finito, de opciones posibles.

Tennankore KK, d'Gama C, Faratro R, Fung S, Wong E, Chan CT. Adverse technical events in home hemodialysis. Am J Kidney Dis. 2015 Jan; 65(1):116-21. doi:10.1053/j.ajkd.2014.08.013. Epub 2014 0ct 16.

Aunque están demostrados los beneficios de la HDD no se debe ignorar el riesgo que supone la realización de un procedimiento complejo fuera de un ambiente hospitalario por el propio paciente y su familia. Por esto, los autores del artículo se planearon como objetivo describir los eventos adversos técnicos en una gran cohorte de pacientes con HDD. Con este fin diseñaron un estudio retrospectivo de los pacientes sometidos a HDD dependientes de un hospital de tercer nivel entre1999 y 2012. Se recogieron todos los acontecimientos adversos graves (definidos como aquellos que requieren la intervención de los profesionales).

Se analizaron los eventos negativos de 202 pacientes con un seguimiento total de 757 pacientes/año. Los pacientes se sometían a una mediana de 5 tratamientos de diálisis por semana y 8 horas por sesión. Se identificaron 22 primeros eventos adversos y 7 eventos recurrentes. Las tasas de eventos adversos fueron 0,049/año provocados por la fístula arteriovenosa, 0.015/año por injerto arteriovenoso, y 0,022/año por catéter de diálisis. Las tasas de eventos por cada 1.000 tratamientos de diálisis fueron 0,208, 0,068, y 0,087 para la fístula arteriovenosa, injerto arteriovenoso, y el catéter, respectivamente. La mayoría de los eventos adversos se relacionan con desplazamiento de la aguja (18) o una embolia gaseosa (6). Requirieron de una visita al servicio de urgencias 8 eventos adversos, de los cuales 5 ingresaron. La tasa de eventos adversos graves fue de 0,009 por paciente/año de HDD y 0.038 por 1.000 tratamientos de diálisis. Los pacien- tes necesitaron 3 transfusiones de sangre, 2 cambios de catéteres, 1 tratamiento con líquidos intravenosos, y una diálisis urgente. Se destaca que se recicló y revisó la técnica en todos los pacientes que presentaron un primer evento adverso.

Aunque se destaca que una de las limitaciones del estudio es que los pacientes no comunicaran eventos negativos menores, la conclusión de este artículo es que los eventos negativos en HDD son relativamente raros y que para preverlos se puede incidir en el reciclaje del paciente y en la revisión periódica del funcionamiento de los accesos vasculares.

$$
\begin{aligned}
& \text { Recibido: } 13 \text { junio } 16 \\
& \text { Revisado: } 15 \text { junio } 16 \\
& \text { Modificado: } 17 \text { junio } 16 \\
& \text { Aceptado: } 21 \text { junio } 16
\end{aligned}
$$

\section{Bibliografía}

1. Andreu L, Force E. 500 Cuestiones que plantea el cuidado del enfermo renal. $2^{\mathrm{a}}$ ed. Barcelona: Masson; 2001.

2. Pérez-Alba $A$, Barril-Cuadrado $G$, Castellano-Cerviño C, Martín-Reyes G, Pérez-Melóne C, Slon-Roblerof $F$, Bajo-Rubio MA. Hemodiálisis domiciliaria en España. Nefrologia.2015;35:1-5.

3. Tennankore K, Nadeau-Fredette AC, Chan CT. Intensified home hemodialysis: Clinical benefits, risks and target populations. Nephrol Dial Transplant 2014; 29: 1342-9.

4. AAVV.Grupo de trabajo sobre Apoyo y Promoción de la Hemodiálisis Domiciliaria en España. SEN Nefrología. Consultado 20/5/16. http://senefro. org $/$ modules.php? name=grupos\&d_op=viewgroup \&idgroup $=12766$ 